\title{
Existence of solutions for a sequential fractional differential system with coupled boundary conditions
}

Jiqiang Jiang ${ }^{1,2^{*}}$ and Lishan Liu²

"Correspondence: qfjjg@163.com 'School of Statistics, Qufu Normal University, Qufu, Shandong 273165 P.R. China

${ }^{2}$ School of Mathematical Sciences, Qufu Normal University, Qufu, Shandong 273165, P.R. China

\section{Springer}

\begin{abstract}
This paper is concerned with the existence and uniqueness of solutions for a sequential fractional differential system with coupled boundary conditions. The existence of solutions is derived by applying Leray-Schauder's alternative, while the uniqueness of the solution is established via Banach's contraction principle. Two examples are then given to demonstrate the validity of our main results.
\end{abstract}

MSC: 26A33; 34B10; 34B15; 34B18

Keywords: fractional differential system; sequential fractional derivative; coupled boundary conditions; fixed point theorem

\section{Introduction}

The human immunodeficiency virus (HIV) causes acquired immune deficiency syndrome (AIDS). The viral infection is characterized by a severe impairment of the immune system and related opportunistic infections. HIV is a retrovirus that targets the $\mathrm{CD} 4^{+} \mathrm{T}$ lymphocytes, which are the most abundant white blood cells of the immune system. Until now, there are several countries, particularly in Africa, with up to $35 \%$ of their populations between the ages of 15 and 50 years infected by HIV, and throughout the world, already over 16 million deaths died of AIDS. Mathematical models have been proven valuable in understanding the dynamics of HIV infection [1-4]. Perelson [5, 6] developed a simple model for the primary infection with HIV. In this model, four categories of cells were defined: uninfected CD $4^{+} \mathrm{T}$-cells, latently infected $\mathrm{CD} 4^{+} \mathrm{T}$-cells, productively infected $\mathrm{CD} 4^{+} \mathrm{T}$-cells, and the virus population. In [4], two equations were proposed to describe the evolution of the system for HIV-1 population dynamics:

$$
\left\{\begin{array}{l}
\frac{d x}{d t}=s-\mu x-\beta x y, \\
\frac{d y}{d t}=\beta x y-\alpha y,
\end{array}\right.
$$

where all parameters and variables are non-negative, and $x$ denotes the number of uninfected CD4 ${ }^{+}$T-cells and $y$ denotes the number of infected cells, $s$ is the assumed constant rate of production of $C D 4^{+}$T-cells, $\mu$ is their per capita death rate, $\beta$ is the rate of infection of CD4 $4^{+}$T-cells by virus, and $\alpha$ is the rate of disappearance of infected cells.

(c) 2016 Jiang and Liu. This article is distributed under the terms of the Creative Commons Attribution 4.0 International License (http://creativecommons.org/licenses/by/4.0/), which permits unrestricted use, distribution, and reproduction in any medium, provided you give appropriate credit to the original author(s) and the source, provide a link to the Creative Commons license, and indicate if changes were made. 
Recently, Arafal et al. [7] introduced fractional-order model of infection of CD4+ T-cells which is described by the following set of FODEs of order $\alpha_{1}, \alpha_{2}, \alpha_{3}>0$ :

$$
\left\{\begin{array}{l}
\mathcal{D}^{\alpha_{1}}(T)=s-K V T-d T+b I, \\
\mathcal{D}^{\alpha_{2}}(I)=K V T-(b+\delta) I, \\
\mathcal{D}^{\alpha_{3}}(V)=N \delta I-c V,
\end{array}\right.
$$

where $T, I$, and $V$ denote the concentration of uninfected $C D 4^{+}$T-cells, infected CD4 ${ }^{+}$ T-cells, and free HIV virus particles in the blood, respectively. $\delta$ represents the death rate of infected T-cells and includes the possibility of death by bursting of infected T-cells, hence $\delta \geq d$. The parameter $b$ is the rate at which infected cells return to the uninfected class, while $c$ is the death rate of the virus and $N$ is the average number of viral particles produced by an infected cell.

Motivated by the HIV infection model and its application background, in this paper, we consider the existence of solutions for the nonlinear sequential fractional differential system with coupled boundary conditions (BCs) of the type:

$$
\begin{cases}\left({ }^{\mathrm{C}} \mathcal{D}^{p}+\lambda_{1}{ }^{\mathrm{C}} \mathcal{D}^{p-1}\right) u(t)=f_{1}(t, u(t), v(t)), & 0<t<1, \\ \left({ }^{\mathrm{c}} \mathcal{D}^{q}+\lambda_{2}{ }^{\mathrm{c}} \mathcal{D}^{q-1}\right) v(t)=f_{2}(t, u(t), v(t)), & 0<t<1, \\ u(0)=u^{\prime}(0)=0, \quad u(1)=a v(\xi), & \\ v(0)=v^{\prime}(0)=0, \quad v(1)=b u(\eta), & \end{cases}
$$

where $\lambda_{i}>0(i=1,2)$ is a parameter, $2<p, q \leq 3,{ }^{\mathrm{c}} \mathcal{D}^{p},{ }^{\mathrm{c}} \mathcal{D}^{q}$ are the Caputo fractional derivatives, $\xi, \eta$ satisfy $\xi, \eta \in(0,1)$ and $a b\left(\lambda_{1} \eta-1+e^{-\lambda_{1} \eta}\right)\left(\lambda_{2} \xi-1+e^{-\lambda_{2} \xi}\right)-\left(\lambda_{1}-1+e^{-\lambda_{1}}\right)\left(\lambda_{2}-1+\right.$ $\left.e^{-\lambda_{2}}\right) \triangleq \Lambda \neq 0$, the nonlinearities $f_{1}, f_{2}:[0,1] \times \mathbb{R} \times \mathbb{R} \rightarrow \mathbb{R}$ are given continuous functions. In the past decades, fractional calculus has been extensively applied in many fields such as physics, chemistry, aerodynamics, electrodynamics of complex medium, polymer rheology, economics, control theory, signal and image processing, biophysics, and blood flow phenomena. Many mathematicians and applied researchers have tried to model real processes using the fractional calculus. In biology, it has been deduced that the membranes of cells of biological organism have fractional-order electrical conductance [8] and thus are classified in groups of non-integer-order models. Fractional derivatives embody essential features of cell rheological behavior and have enjoyed greatest success in the field of rheology [9]. Fractional-order ordinary differential equations are naturally related to systems with long time memory which exists in most biological systems such as HIV infection, hepatitis $\mathrm{C}$ virus $(\mathrm{HCV})$ infection, and cancer pervasion. Also, they are closely related to fractals, which are abundant in biological systems. Thus fractional-order differential equations are also regarded as a better tool for the description of hereditary properties of various materials and processes than the corresponding integer-order differential equations. With this advantage, fractional-order models have become more realistic and practical than the corresponding classical integer-order models, moreover, the dynamics behavior of fractional-order models are also as stable as their integer-order counterpart. Since theoretical results can help to get an in-depth understanding for the dynamic behavior in biological process, the study of abstract fractional dynamic models nowadays is quite relevant and important. On the other hand, BCs in (1.1) are referred to as coupled BCs; they arise in the study of reaction-diffusion equations, Sturm-Liouville problems, mathematical biology and so on; see [10-20]. In [16], Leung studied the following reaction-diffusion 
system for a prey-predator interaction:

$$
\left\{\begin{array}{l}
u_{t}(t, x)=\sigma_{1} \Delta u+u(a+f(u, v)), \quad t \geq 0, x \in \Omega \subset \mathbb{R}^{n}, \\
v_{t}(t, x)=\sigma_{2} \Delta v+v(-r+g(u, v)), \quad t \geq 0, x \in \Omega \subset \mathbb{R}^{n},
\end{array}\right.
$$

subject to the coupled BCs

$$
\frac{\partial u}{\partial \eta}=0, \quad \frac{\partial v}{\partial \eta}-p(u)-q(v)=0 \quad \text { on } \partial \Omega
$$

where the functions $u(t, x), v(t, x)$, respectively, represent the density of prey and predator at time $t \geq 0$ and at position $x=\left(x_{1}, \ldots, x_{n}\right)$. Similar coupled BCs are also studied in [11] for a biochemical system. As far as we know, the nonlinear fractional differential system coupled at equations have been studied extensively. For details, see [21-31] and the references therein. However, there have been a few papers which deal with coupling at boundary conditions for fractional differential systems. Wang et al. [32] obtained the existence and uniqueness of positive solution to nonzero boundary values problem for a coupled system of nonlinear fractional differential equations,

$$
\left\{\begin{array}{l}
\mathcal{D}_{0+}^{\alpha} u(t)=f(t, v(t)), \quad \mathcal{D}_{0+}^{\beta} v(t)=g(t, u(t)), \quad 0<t<1, \\
u(0)=v(0)=0, \quad u(1)=a u(\xi), \quad v(1)=b v(\xi),
\end{array}\right.
$$

where $1<\alpha, \beta<2,0 \leq a, b<1,0<\xi<1, f, g:[0,1] \times[0,+\infty) \rightarrow[0,+\infty)$ are continuous functions, and $\mathcal{D}_{0_{+}}^{\alpha}, \mathcal{D}_{0_{+}}^{\beta}$ are the standard Riemann-Liouville fractional derivative.

The paper is organized as follows. In Section 2, we recall some basic concepts of fractional calculus and present some auxiliary lemmas. The main results are presented in Section 3. We give two results: the first one dealing with the existence of solutions is derived by applying Leray-Schauder's alternative; the second one concerning the uniqueness of solutions, established by applying Banach's contraction mapping principle. In Section 4, two examples are given to demonstrate the validity of our main results. Some interesting observations are presented in the conclusions section.

\section{Preliminaries and lemmas}

In this section, we will present some preliminaries and lemmas that will be used in the proof of our main results.

Definition 2.1 ([33, 34] $)$ The Riemann-Liouville fractional integral of order $\alpha>0$ is given by

$$
I^{\alpha} u(t)=\frac{1}{\Gamma(\alpha)} \int_{0}^{t}(t-s)^{\alpha-1} u(s) d s
$$

where $n-1<\alpha<n$, provided that the right-hand side is pointwise defined on $(0,+\infty)$.

Definition $2.2([33,34])$ For an $(n-1)$-times absolutely continuous function $u:[0, \infty) \rightarrow$ $\mathbb{R}$, the Caputo derivative of fractional order $\alpha$ is defined as

$$
{ }^{\mathrm{c}} \mathcal{D}^{\alpha} u(t)=\frac{1}{\Gamma(n-\alpha)} \int_{0}^{t}(t-s)^{n-\alpha-1} u^{(n)}(s) d s, \quad n-1<\alpha<n, n=[\alpha]+1,
$$

where $[\alpha]$ denotes the integer part of the real number $\alpha$. 
Lemma 2.1 For any $h, g \in L(0,1) \cap C(0,1)$, the system consisting of the equations

$$
\left({ }^{\mathrm{c}} \mathcal{D}^{p}+\lambda_{1}{ }^{\mathrm{c}} \mathcal{D}^{p-1}\right) u(t)=h(t), \quad\left({ }^{\mathrm{c}} \mathcal{D}^{q}+\lambda_{2}{ }^{\mathrm{c}} \mathcal{D}^{q-1}\right) v(t)=g(t), \quad t \in(0,1)
$$

and the $B C s$

$$
\begin{cases}u(0)=u^{\prime}(0)=0, & u(1)=a v(\xi), \\ v(0)=v^{\prime}(0)=0, & v(1)=b u(\eta),\end{cases}
$$

has a unique integral representation

$$
\begin{aligned}
u(t)= & A_{1}(t)\left\{a\left(\lambda_{2} \xi-1+e^{-\lambda_{2} \xi}\right)\left[\int_{0}^{1} e^{-\lambda_{2}(1-s)}(Q g)(s) d s-b \int_{0}^{\eta} e^{-\lambda_{1}(\eta-s)}(P h)(s) d s\right]\right. \\
& \left.-\left(\lambda_{2}-1+e^{-\lambda_{2}}\right)\left[a \int_{0}^{\xi} e^{-\lambda_{2}(\xi-s)}(Q g)(s) d s-\int_{0}^{1} e^{-\lambda_{1}(1-s)}(P h)(s) d s\right]\right\} \\
& +\int_{0}^{t} e^{-\lambda_{1}(t-s)}(P h)(s) d s, \\
v(t)= & A_{2}(t)\left\{\left(\lambda_{1}-1+e^{-\lambda_{1}}\right)\left[\int_{0}^{1} e^{-\lambda_{2}(1-s)}(Q g)(s) d s-b \int_{0}^{\eta} e^{-\lambda_{1}(\eta-s)}(P h)(s) d s\right]\right. \\
& \left.-b\left(\lambda_{1} \eta-1+e^{-\lambda_{1} \eta}\right)\left[a \int_{0}^{\xi} e^{-\lambda_{2}(\xi-s)}(Q g)(s) d s-\int_{0}^{1} e^{-\lambda_{1}(1-s)}(P h)(s) d s\right]\right\} \\
& +\int_{0}^{t} e^{-\lambda_{2}(t-s)}(Q g)(s) d s,
\end{aligned}
$$

where

$$
\begin{aligned}
& A_{1}(t)=\frac{1}{\Lambda}\left(\lambda_{1} t-1+e^{-\lambda_{1} t}\right), \quad A_{2}(t)=\frac{1}{\Lambda}\left(\lambda_{2} t-1+e^{-\lambda_{2} t}\right), \\
& (P h)(s)=\int_{0}^{s} \frac{(s-\tau)^{p-2}}{\Gamma(p-1)} h(\tau) d \tau, \quad(Q g)(s)=\int_{0}^{s} \frac{(s-\tau)^{q-2}}{\Gamma(q-1)} g(\tau) d \tau .
\end{aligned}
$$

Proof Solving (2.1), we obtain

$$
\begin{aligned}
& u(t)=c_{11} e^{-\lambda_{1} t}+\frac{c_{12}}{\lambda_{1}}\left(1-e^{-\lambda_{1} t}\right)+\frac{c_{13}}{\lambda_{1}^{2}}\left(\lambda_{1} t-1+e^{-\lambda_{1} t}\right)+\int_{0}^{t} e^{-\lambda_{1}(t-s)}(P h)(s) d s, \\
& v(t)=c_{21} e^{-\lambda_{2} t}+\frac{c_{22}}{\lambda_{2}}\left(1-e^{-\lambda_{2} t}\right)+\frac{c_{23}}{\lambda_{2}^{2}}\left(\lambda_{2} t-1+e^{-\lambda_{2} t}\right)+\int_{0}^{t} e^{-\lambda_{2}(t-s)}(Q g)(s) d s,
\end{aligned}
$$

where $c_{i j}(1 \leq i \leq 2,1 \leq j \leq 3)$ are constants to be determined. In the following, we determine $c_{i j}(1 \leq i \leq 2,1 \leq j \leq 3)$, so that $u(t)$ and $v(t)$ satisfy (2.2). By BCs (2.2), we obtain

$$
c_{11}=c_{12}=0, \quad c_{21}=c_{22}=0,
$$

and

$$
\begin{aligned}
& \frac{\lambda_{1}-1+e^{-\lambda_{1}}}{\lambda_{1}^{2}} c_{13}-\frac{a\left(\lambda_{2} \xi-1+e^{-\lambda_{2} \xi}\right)}{\lambda_{2}^{2}} c_{23} \\
& =a \int_{0}^{\xi} e^{-\lambda_{2}(\xi-s)}(Q g)(s) d s-\int_{0}^{1} e^{-\lambda_{1}(1-s)}(P h)(s) d s,
\end{aligned}
$$




$$
\begin{aligned}
& \frac{b\left(\lambda_{1} \eta-1+e^{-\lambda_{1} \eta}\right)}{\lambda_{1}^{2}} c_{13}-\frac{\lambda_{2}-1+e^{-\lambda_{2}}}{\lambda_{2}^{2}} c_{23} \\
& \quad=\int_{0}^{1} e^{-\lambda_{2}(1-s)}(Q g)(s) d s-b \int_{0}^{\eta} e^{-\lambda_{1}(\eta-s)}(P h)(s) d s .
\end{aligned}
$$

Note that

$$
\begin{aligned}
\left|\begin{array}{rr}
\frac{\lambda_{1}-1+e^{-\lambda_{1}}}{\lambda_{1}^{2}} & -\frac{a\left(\lambda_{2} \xi-1+e^{-\lambda_{2} \xi}\right)}{\lambda_{2}^{2}} \\
\frac{b\left(\lambda_{1} \eta-1+e^{-\lambda_{1} \eta}\right)}{\lambda_{1}^{2}} & -\frac{\lambda_{2}-1+e^{-\lambda_{2}}}{\lambda_{2}^{2}}
\end{array}\right|= & \frac{a b\left(\lambda_{1} \eta-1+e^{-\lambda_{1} \eta}\right)\left(\lambda_{2} \xi-1+e^{-\lambda_{2} \xi}\right)}{\lambda_{1}^{2} \lambda_{2}^{2}} \\
& -\frac{\left(\lambda_{1}-1+e^{-\lambda_{1}}\right)\left(\lambda_{2}-1+e^{-\lambda_{2}}\right)}{\lambda_{1}^{2} \lambda_{2}^{2}}=\frac{\Lambda}{\lambda_{1}^{2} \lambda_{2}^{2}} \triangleq \mathfrak{B} \neq 0 .
\end{aligned}
$$

Thus, the system (2.10)-(2.11) has a unique solution for $c_{13}$ and $c_{23}$. By Cramer's rule and simple calculations, it follows that

$$
\begin{aligned}
c_{13}= & \frac{1}{\mathfrak{B}} \frac{a\left(\lambda_{2} \xi-1+e^{-\lambda_{2} \xi}\right)}{\lambda_{2}^{2}}\left[\int_{0}^{1} e^{-\lambda_{2}(1-s)}(Q g)(s) d s-b \int_{0}^{\eta} e^{-\lambda_{1}(\eta-s)}(P h)(s) d s\right] \\
& -\frac{1}{\mathfrak{B}} \frac{\lambda_{2}-1+e^{-\lambda_{2}}}{\lambda_{2}^{2}}\left[a \int_{0}^{\xi} e^{-\lambda_{2}(\xi-s)}(Q g)(s) d s-\int_{0}^{1} e^{-\lambda_{1}(1-s)}(P h)(s) d s\right], \\
c_{23}= & \frac{1}{\mathfrak{B}} \frac{\lambda_{1}-1+e^{-\lambda_{1}}}{\lambda_{1}^{2}}\left[\int_{0}^{1} e^{-\lambda_{2}(1-s)}(Q g)(s) d s-b \int_{0}^{\eta} e^{-\lambda_{1}(\eta-s)}(P h)(s) d s\right] \\
& -\frac{1}{\mathfrak{B}} \frac{b\left(\lambda_{1} \eta-1+e^{-\lambda_{1} \eta}\right)}{\lambda_{1}^{2}}\left[a \int_{0}^{\xi} e^{-\lambda_{2}(\xi-s)}(Q g)(s) d s-\int_{0}^{1} e^{-\lambda_{1}(1-s)}(P h)(s) d s\right] .
\end{aligned}
$$

Substituting (2.9) and (2.12) in (2.7), one has

$$
\begin{aligned}
u(t)= & A_{1}(t)\left\{a\left(\lambda_{2} \xi-1+e^{-\lambda_{2} \xi}\right)\left[\int_{0}^{1} e^{-\lambda_{2}(1-s)}(Q g)(s) d s-b \int_{0}^{\eta} e^{-\lambda_{1}(\eta-s)}(P h)(s) d s\right]\right. \\
& \left.-\left(\lambda_{2}-1+e^{-\lambda_{2}}\right)\left[a \int_{0}^{\xi} e^{-\lambda_{2}(\xi-s)}(Q g)(s) d s-\int_{0}^{1} e^{-\lambda_{1}(1-s)}(P h)(s) d s\right]\right\} \\
& +\int_{0}^{t} e^{-\lambda_{1}(t-s)}(P h)(s) d s .
\end{aligned}
$$

So (2.3) holds. Similarly, substituting (2.9) and (2.13) in (2.8) we can get (2.4). This completes the proof of the lemma.

Lemma $2.2([35])$ For any $h, g \in L(0,1) \cap C(0,1)$, we have

$$
\begin{aligned}
& \left|\int_{0}^{\eta} e^{-\lambda_{1}(\eta-s)}(P h)(s) d s\right| \leq \frac{\eta^{p}}{\lambda_{1} \Gamma(p)}\left(1-e^{-\lambda_{1} \eta}\right)\|h\|, \\
& \left|\int_{0}^{t} e^{-\lambda_{1}(t-s)}(P h)(s) d s\right| \leq \frac{1}{\lambda_{1} \Gamma(p)}\left(1-e^{-\lambda_{1}}\right)\|h\|, \\
& \left|\int_{0}^{\xi} e^{-\lambda_{2}(\xi-s)}(Q g)(s) d s\right| \leq \frac{\xi^{q}}{\lambda_{2} \Gamma(q)}\left(1-e^{-\lambda_{2} \xi}\right)\|g\|, \\
& \left|\int_{0}^{t} e^{-\lambda_{2}(t-s)}(Q g)(s) d s\right| \leq \frac{1}{\lambda_{2} \Gamma(q)}\left(1-e^{-\lambda_{2}}\right)\|g\| .
\end{aligned}
$$


Let $X=C[0,1]$, then $X \times X$ is a Banach space with the norm

$$
\|(u, v)\|_{1}:=\|u\|+\|v\|, \quad\|u\|=\max _{0 \leq t \leq 1}|u(t)|, \quad\|v\|=\max _{0 \leq t \leq 1}|v(t)|,
$$

for any $(u, v) \in X \times X$.

In view of Lemma 2.1, we define the operator $T: X \times X \rightarrow X \times X$ by

$$
T(u, v)=\left(T_{1}(u, v), T_{2}(u, v)\right),
$$

where operators $T_{i}: X \times X \rightarrow X(i=1,2)$ are defined by

$$
\begin{aligned}
& T_{1}(u, v)(t) \\
& =A_{1}(t)\left\{a\left(\lambda_{2} \xi-1+e^{-\lambda_{2} \xi}\right)\left[\int_{0}^{1} e^{-\lambda_{2}(1-s)} Q(u, v)(s) d s-b \int_{0}^{\eta} e^{-\lambda_{1}(\eta-s)} P(u, v)(s) d s\right]\right. \\
& \left.\quad-\left(\lambda_{2}-1+e^{-\lambda_{2}}\right)\left[a \int_{0}^{\xi} e^{-\lambda_{2}(\xi-s)} Q(u, v)(s) d s-\int_{0}^{1} e^{-\lambda_{1}(1-s)} P(u, v)(s) d s\right]\right\} \\
& \quad+\int_{0}^{t} e^{-\lambda_{1}(t-s)} P(u, v)(s) d s
\end{aligned}
$$

and

$$
\begin{aligned}
T_{2}(u, v)(t) & \\
= & A_{2}(t)\left\{\left(\lambda_{1}-1+e^{-\lambda_{1}}\right)\left[\int_{0}^{1} e^{-\lambda_{2}(1-s)} Q(u, v)(s) d s-b \int_{0}^{\eta} e^{-\lambda_{1}(\eta-s)} P(u, v)(s) d s\right]\right. \\
& \left.-b\left(\lambda_{1} \eta-1+e^{-\lambda_{1} \eta}\right)\left[a \int_{0}^{\xi} e^{-\lambda_{2}(\xi-s)} Q(u, v)(s) d s-\int_{0}^{1} e^{-\lambda_{1}(1-s)} P(u, v)(s) d s\right]\right\} \\
& +\int_{0}^{t} e^{-\lambda_{2}(t-s)} Q(u, v)(s) d s,
\end{aligned}
$$

with

$$
\begin{aligned}
& P(u, v)(s)=\int_{0}^{s} \frac{(s-\tau)^{p-2}}{\Gamma(p-1)} f_{1}(\tau, u(\tau), v(\tau)) d \tau, \\
& Q(u, v)(s)=\int_{0}^{s} \frac{(s-\tau)^{q-2}}{\Gamma(q-1)} f_{2}(\tau, u(\tau), v(\tau)) d \tau .
\end{aligned}
$$

For the sake of convenience, we set

$$
\begin{aligned}
& A_{1}=\sup _{t \in[0,1]}\left|A_{1}(t)\right|, \quad A_{2}=\sup _{t \in[0,1]}\left|A_{2}(t)\right| \\
& M_{1}=\frac{A_{1}\left[|a b|\left(\lambda_{2} \xi-1+e^{-\lambda_{2} \xi}\right)\left(1-e^{-\lambda_{1} \eta}\right) \eta^{p-1}+\left(\lambda_{2}-1+e^{-\lambda_{2}}\right)\left(1-e^{-\lambda_{1}}\right)\right]+\left(1-e^{-\lambda_{1}}\right)}{\lambda_{1} \Gamma(p)} \\
& M_{2}=\frac{A_{1}\left[|a|\left(\lambda_{2} \xi-1+e^{-\lambda_{2} \xi}\right)\left(1-e^{-\lambda_{2}}\right)+\left(\lambda_{2}-1+e^{-\lambda_{2}}\right)|a|\left(1-e^{-\lambda_{2} \xi}\right) \xi^{q-1}\right]}{\lambda_{2} \Gamma(q)} \\
& M_{1}^{\prime}=\frac{A_{2}\left[\left(\lambda_{1}-1+e^{-\lambda_{1}}\right)|b|\left(1-e^{-\lambda_{1} \eta}\right) \eta^{p-1}+|b|\left(\lambda_{1} \eta-1+e^{-\lambda_{1} \eta}\right)\left(1-e^{-\lambda_{1}}\right)\right]}{\lambda_{1} \Gamma(p)}
\end{aligned}
$$




$$
M_{2}^{\prime}=\frac{A_{2}\left[\left(\lambda_{1}-1+e^{-\lambda_{1}}\right)\left(1-e^{-\lambda_{2}}\right)+|a b|\left(\lambda_{1} \eta-1+e^{-\lambda_{1} \eta}\right)\left(1-e^{-\lambda_{2} \xi}\right) \xi^{q-1}\right]+\left(1-e^{-\lambda_{2}}\right)}{\lambda_{2} \Gamma(q)}
$$

and

$$
(P \mathbf{1})(s)=\int_{0}^{s} \frac{(s-\tau)^{p-2}}{\Gamma(p-1)} d \tau, \quad(Q \mathbf{1})(s)=\int_{0}^{s} \frac{(s-\tau)^{q-2}}{\Gamma(q-1)} d \tau .
$$

Lemma 2.3 The operator $T: X \times X \rightarrow X \times X$ is a completely continuous.

Proof By continuity of the functions $f_{i}(i=1,2)$, the operator $T$ is continuous.

Let $\Omega \subset X \times X$ be bounded. Then there exist constants $L_{i}>0(i=1,2)$ such that

$$
\left|f_{i}(t, u(t), v(t))\right| \leq L_{i}, \quad \forall(u, v) \in \Omega, i=1,2 .
$$

Then for any $(u, v) \in \Omega$, it follows from (2.16), (2.14), (2.15), (2.20), and (2.22) that

$$
\begin{aligned}
\left|T_{1}(u, v)(t)\right| & \\
\leq & A_{1}\left\{|a|\left(\lambda_{2} \xi-1+e^{-\lambda_{2} \xi}\right)\left[L_{2} \int_{0}^{1} e^{-\lambda_{2}(1-s)}(Q \mathbf{1})(s) d s+|b| L_{1} \int_{0}^{\eta} e^{-\lambda_{1}(\eta-s)}(P \mathbf{1})(s) d s\right]\right. \\
& \left.+\left(\lambda_{2}-1+e^{-\lambda_{2}}\right)\left[|a| L_{2} \int_{0}^{\xi} e^{-\lambda_{2}(\xi-s)}(Q \mathbf{1})(s) d s+L_{1} \int_{0}^{1} e^{-\lambda_{1}(1-s)}(P \mathbf{1})(s) d s\right]\right\} \\
& +L_{1} \int_{0}^{t} e^{-\lambda_{1}(t-s)}(P \mathbf{1})(s) d s \\
\leq & A_{1}\left[|a|\left(\lambda_{2} \xi-1+e^{-\lambda_{2} \xi}\right)\left(\frac{L_{2}\left(1-e^{-\lambda_{2}}\right)}{\lambda_{2} \Gamma(q)}+\frac{|b| L_{1}\left(1-e^{-\lambda_{1} \eta}\right) \eta^{p-1}}{\lambda_{1} \Gamma(p)}\right)\right. \\
& \left.+\left(\lambda_{2}-1+e^{-\lambda_{2}}\right)\left(\frac{|a| L_{2}\left(1-e^{-\lambda_{2} \xi}\right) \xi^{q-1}}{\lambda_{2} \Gamma(q)}+\frac{L_{1}\left(1-e^{-\lambda_{1}}\right)}{\lambda_{1} \Gamma(p)}\right)\right] \\
& +\frac{L_{1}\left(1-e^{-\lambda_{1}}\right)}{\lambda_{1} \Gamma(p)} \\
\leq & L_{1} \frac{A_{1}\left[|a b|\left(\lambda_{2} \xi-1+e^{-\lambda_{2} \xi}\right)\left(1-e^{-\lambda_{1} \eta}\right) \eta^{p-1}+\left(\lambda_{2}-1+e^{-\lambda_{2}}\right)\left(1-e^{-\lambda_{1}}\right)\right]+\left(1-e^{-\lambda_{1}}\right)}{\lambda_{1} \Gamma(p)} \\
& +L_{2} \frac{A_{1}\left[|a|\left(\lambda_{2} \xi-1+e^{-\lambda_{2} \xi}\right)\left(1-e^{-\lambda_{2}}\right)+\left(\lambda_{2}-1+e^{-\lambda_{2}}\right)|a|\left(1-e^{-\lambda_{2} \xi}\right) \xi^{q-1}\right]}{\lambda_{2} \Gamma(q)}, \quad(2.23)
\end{aligned}
$$

which implies that

$$
\left\|T_{1}(u, v)\right\| \leq L_{1} M_{1}+L_{2} M_{2}
$$

where $M_{1}, M_{2}$ are given by (2.21).

By (2.17), (2.14), (2.15), (2.20), (2.22), and proceeding as in (2.23), we can obtain

$$
\left\|T_{2}(u, v)\right\| \leq L_{1} M_{1}^{\prime}+L_{2} M_{2}^{\prime}
$$

where $M_{1}^{\prime}, M_{2}^{\prime}$ are given by (2.21). 
Combining (2.24) with(2.25), we obtain

$$
\|T(u, v)\|_{1}=\left\|T_{1}(u, v)\right\|+\left\|T_{2}(u, v)\right\| \leq\left(L_{1} M_{1}+L_{2} M_{2}\right)+\left(L_{1} M_{1}^{\prime}+L_{2} M_{2}^{\prime}\right)=M,
$$

which implies that the operator $T$ is uniformly bounded.

Next, we show that $T$ is equicontinuous. For any $t_{1}, t_{2} \in[0,1]$ with $t_{1} \leq t_{2}$, noticing (2.22) then we have

$$
\begin{aligned}
& \left|T_{1}(u, v)\left(t_{2}\right)-T_{1}(u, v)\left(t_{1}\right)\right| \\
& \leq \mid\left[A_{1}\left(t_{2}\right)-A_{1}\left(t_{1}\right)\right]\left\{a\left(\lambda_{2} \xi-1+e^{-\lambda_{2} \xi}\right)\right. \\
& \quad \times\left[L_{2} \int_{0}^{1} e^{-\lambda_{2}(1-s)}(Q \mathbf{1})(s) d s-b L_{1} \int_{0}^{\eta} e^{-\lambda_{1}(\eta-s)}(P \mathbf{1})(s) d s\right] \\
& \left.\quad-\left(\lambda_{2}-1+e^{-\lambda_{2}}\right)\left[a L_{2} \int_{0}^{\xi} e^{-\lambda_{2}(\xi-s)}(Q \mathbf{1})(s) d s-L_{1} \int_{0}^{1} e^{-\lambda_{1}(1-s)}(P \mathbf{1})(s) d s\right]\right\} \mid \\
& \quad+L_{1}\left|\int_{0}^{t_{1}}\left(e^{-\lambda_{1}\left(t_{2}-s\right)}-e^{-\lambda_{1}\left(t_{1}-s\right)}\right)(P \mathbf{1})(s) d s+\int_{t_{1}}^{t_{2}} e^{-\lambda_{1}\left(t_{2}-s\right)}(P \mathbf{1})(s) d s\right|
\end{aligned}
$$

Analogously, we can obtain the following inequalities:

$$
\begin{aligned}
& \left|T_{2}(u, v)\left(t_{2}\right)-T_{2}(u, v)\left(t_{1}\right)\right| \\
& \leq \mid\left[A_{2}\left(t_{2}\right)-A_{2}\left(t_{1}\right)\right]\left\{\left(\lambda_{1}-1+e^{-\lambda_{1}}\right)\right. \\
& \quad \times\left[L_{2} \int_{0}^{1} e^{-\lambda_{2}(1-s)}(Q \mathbf{1})(s) d s-b L_{1} \int_{0}^{\eta} e^{-\lambda_{1}(\eta-s)}(P \mathbf{1})(s) d s\right] \\
& \left.\quad-b\left(\lambda_{1} \eta-1+e^{-\lambda_{1} \eta}\right)\left[a L_{2} \int_{0}^{\xi} e^{-\lambda_{2}(\xi-s)}(Q \mathbf{1})(s) d s-L_{1} \int_{0}^{1} e^{-\lambda_{1}(1-s)}(P \mathbf{1})(s) d s\right]\right\} \mid \\
& \quad+L_{2}\left|\int_{0}^{t_{1}}\left(e^{-\lambda_{2}\left(t_{2}-s\right)}-e^{-\lambda_{2}\left(t_{1}-s\right)}\right)(Q \mathbf{1})(s) d s+\int_{t_{1}}^{t_{2}} e^{-\lambda_{2}\left(t_{2}-s\right)}(Q \mathbf{1})(s) d s\right| .
\end{aligned}
$$

Since for any fixed $s \in[0,1]$, the functions $e^{-\lambda_{i}(t-s)}, A_{i}(t)(i=1,2)$ are uniformly continuous on the interval on $[0,1]$, we can conclude that the operator $T(u, v)$ is equicontinuous. Thus the operator $T(u, v)$ is completely continuous. The proof is completed.

Now we state a well-known fixed point theorem, which is needed to prove the existence of solutions for system (1.1).

Lemma 2.4 (Leray-Schauder alternative [36]) Let E be a Banach space. Assume that T: $E \rightarrow E$ be a completely continuous operator. Let

$$
V=\{x \in E \mid x=\mu T x \text { for some } 0<\mu<1\} .
$$

Then either the set $V$ is unbounded, or $T$ has at least one fixed point. 


\section{Main results}

Theorem 3.1 Assume that there exist real constants $\rho_{i}, \delta_{i} \geq 0$ and $k_{i}>0(i=1,2)$ such that $\forall t \in[0,1], x, y \in \mathbb{R}$,

$$
\left|f_{i}(t, x, y)\right| \leq k_{i}+\rho_{i}|x|+\delta_{i}|y|, \quad i=1,2
$$

In addition, assume that

$$
\left(M_{1}+M_{1}^{\prime}\right) \rho_{1}+\left(M_{2}+M_{2}^{\prime}\right) \rho_{2}<1 \text { and }\left(M_{1}+M_{1}^{\prime}\right) \delta_{1}+\left(M_{2}+M_{2}^{\prime}\right) \delta_{2}<1 \text {, }
$$

where $M_{i}, M_{i}^{\prime}(i=1,2)$ are defined by (2.21). Then the system (1.1) has at least one solution.

Proof Let us verify that the set $V=\{(u, v) \in X \times X:(u, v)=\mu T(u, v), 0 \leq \mu \leq 1\}$ is bounded. Let $(u, v) \in V$, then $(u, v)=\mu T(u, v)$. For any $t \in[0,1]$, we have

$$
u(t)=\mu T_{1}(u, v)(t), \quad v(t)=\mu T_{2}(u, v)(t) .
$$

Then, by (3.1), (3.2), (2.14), and (2.15), for any $t \in[0,1]$, we have

$$
\begin{aligned}
|u(t)| \leq & \mid A_{1}(t)\left\{a\left(\lambda_{2} \xi-1+e^{-\lambda_{2} \xi}\right)\right. \\
& \times\left[\int_{0}^{1} e^{-\lambda_{2}(1-s)} Q(u, v)(s) d s-b \int_{0}^{\eta} e^{-\lambda_{1}(\eta-s)} P(u, v)(s) d s\right] \\
& \left.-\left(\lambda_{2}-1+e^{-\lambda_{2}}\right)\left[a \int_{0}^{\xi} e^{-\lambda_{2}(\xi-s)} Q(u, v)(s) d s-\int_{0}^{1} e^{-\lambda_{1}(1-s)} P(u, v)(s) d s\right]\right\} \\
& +\int_{0}^{t} e^{-\lambda_{1}(t-s)} P(u, v)(s) d s \mid \\
\leq & A_{1}\left\{| a | ( \lambda _ { 2 } \xi - 1 + e ^ { - \lambda _ { 2 } \xi } ) \left[\left(k_{2}+\rho_{2}\|u\|+\delta_{2}\|v\|\right) \int_{0}^{1} e^{-\lambda_{2}(1-s)}(Q \mathbf{1})(s) d s\right.\right. \\
& \left.+|b|\left(k_{1}+\rho_{1}\|u\|+\delta_{1}\|v\|\right) \int_{0}^{\eta} e^{-\lambda_{1}(\eta-s)}(P \mathbf{1})(s) d s\right] \\
& +\left(\lambda_{2}-1+e^{-\lambda_{2}}\right)\left[|a|\left(k_{2}+\rho_{2}\|u\|+\delta_{2}\|v\|\right) \int_{0}^{\xi} e^{-\lambda_{2}(\xi-s)}(Q \mathbf{1})(s) d s\right. \\
& \left.\left.+\left(k_{1}+\rho_{1}\|u\|+\delta_{1}\|v\|\right) \int_{0}^{1} e^{-\lambda_{1}(1-s)}(P \mathbf{1})(s) d s\right]\right\} \\
& +\left(k_{1}+\rho_{1}\|u\|+\delta_{1}\|v\|\right) \int_{0}^{t} e^{-\lambda_{1}(t-s)}(P \mathbf{1})(s) d s \\
\leq & A_{1}\left[|a b|\left(\lambda_{2} \xi-1+e^{-\lambda_{2} \xi}\right)\left(1-e^{-\lambda_{1} \eta}\right) \eta^{p-1}+\left(\lambda_{2}-1+e^{-\lambda_{2}}\right)\left(1-e^{-\lambda_{1}}\right)\right]+\left(1-e^{-\lambda_{1}}\right) \\
& \times\left(k_{1} \Gamma(p)\right. \\
& \times \frac{A_{1}\left[|a|\left(\lambda_{2} \xi-1\|u\|+\delta_{1}\|v\|\right)+M_{2}\left(k_{2}+\rho_{2}\|u\|+\delta_{1}\|v\|\right)+\left(k_{2}+\rho_{2}\|u\|+\delta_{2}\|v\|\right)\right.}{\left.\left.-\lambda_{2} \xi\right)\left(1-e^{-\lambda_{2}}\right)+\left(\lambda_{2}-1+e^{-\lambda_{2}}\right)|a|\left(1-e^{-\lambda_{2} \xi}\right) \xi^{q-1}\right]} \\
\lambda_{2} \Gamma(q) & (3.3) \\
& \\
&
\end{aligned}
$$


Hence we have

$$
\|u\| \leq M_{1}\left(k_{1}+\rho_{1}\|u\|+\delta_{1}\|v\|\right)+M_{2}\left(k_{2}+\rho_{2}\|u\|+\delta_{2}\|v\|\right) .
$$

Similarly, proceeding as in (3.3), we can obtain

$$
\|v\| \leq M_{1}^{\prime}\left(k_{1}+\rho_{1}\|u\|+\delta_{1}\|v\|\right)+M_{2}^{\prime}\left(k_{2}+\rho_{2}\|u\|+\delta_{2}\|v\|\right) .
$$

Combining (3.4) with (3.5), we obtain

$$
\begin{aligned}
\|u\|+\|v\| \leq & {\left[\left(M_{1}+M_{1}^{\prime}\right) k_{1}+\left(M_{2}+M_{2}^{\prime}\right) k_{2}\right]+\left[\left(M_{1}+M_{1}^{\prime}\right) \rho_{1}+\left(M_{2}+M_{2}^{\prime}\right) \rho_{2}\right]\|u\| } \\
& +\left[\left(M_{1}+M_{1}^{\prime}\right) \delta_{1}+\left(M_{2}+M_{2}^{\prime}\right) \delta_{2}\right]\|v\| .
\end{aligned}
$$

Consequently,

$$
\|(u, v)\|_{1}=\|u\|+\|v\| \leq \frac{\left(M_{1}+M_{1}^{\prime}\right) k_{1}+\left(M_{2}+M_{2}^{\prime}\right) k_{2}}{M_{0}}
$$

where $M_{0}=\min \left\{1-\left[\left(M_{1}+M_{1}^{\prime}\right) \rho_{1}+\left(M_{2}+M_{2}^{\prime}\right) \rho_{2}\right], 1-\left[\left(M_{1}+M_{1}^{\prime}\right) \delta_{1}+\left(M_{2}+M_{2}^{\prime}\right) \delta_{2}\right]\right\}$, which proves that the set $V$ is bounded. Thus, by Lemma 2.4, the operator $T$ has at least one fixed point. Hence the system (1.1) has at least one solution. The proof is complete.

Theorem 3.2 Assume that $f_{i}:[0,1] \times \mathbb{R}^{2} \rightarrow \mathbb{R}$ are continuous functions and there exist constants $m_{i}, n_{i} \geq 0(i=1,2)$, such that $\forall t \in[0,1], x_{i}, y_{i} \in \mathbb{R}$,

$$
\left|f_{i}\left(t, x_{1}, y_{1}\right)-f_{i}\left(t, x_{2}, y_{2}\right)\right| \leq m_{i}\left|x_{1}-x_{2}\right|+n_{i}\left|y_{1}-y_{2}\right|, \quad i=1,2 \text {. }
$$

In addition, assume that

$$
\left(M_{1}+M_{1}^{\prime}\right)\left(m_{1}+n_{1}\right)+\left(M_{2}+M_{2}^{\prime}\right)\left(m_{2}+n_{2}\right)<1,
$$

where $M_{i}, M_{i}^{\prime}(i=1,2)$ are defined by (2.21). Then the system (1.1) has a unique solution.

Proof Define $\sup _{t \in[0,1]}\left|f_{i}(t, 0,0)\right|=N_{i}<\infty(i=1,2)$ such that

$$
r \geq \frac{\left(M_{1}+M_{1}^{\prime}\right) N_{1}+\left(M_{2}+M_{2}^{\prime}\right) N_{2}}{1-\left(M_{1}+M_{1}^{\prime}\right)\left(m_{1}+n_{1}\right)-\left(M_{2}+M_{2}^{\prime}\right)\left(m_{2}+n_{2}\right)}
$$

We show that $T B_{r} \subset B_{r}$, where $B_{r}=\left\{(u, v) \in X \times X:\|(u, v)\|_{1}<r\right\}$. For any $(u, v) \in B_{r}$, we have

$$
\begin{aligned}
& \left|T_{1}(u, v)(t)\right| \\
& \leq \max _{t \in[0,1]} \mid A_{1}(t)\left\{a\left(\lambda_{2} \xi-1+e^{-\lambda_{2} \xi}\right)\right. \\
& \quad \times\left[\int_{0}^{1} e^{-\lambda_{2}(1-s)} Q(u, v)(s) d s-b \int_{0}^{\eta} e^{-\lambda_{1}(\eta-s)} P(u, v)(s) d s\right]
\end{aligned}
$$




$$
\begin{aligned}
& \left.-\left(\lambda_{2}-1+e^{-\lambda_{2}}\right)\left[a \int_{0}^{\xi} e^{-\lambda_{2}(\xi-s)} Q(u, v)(s) d s-\int_{0}^{1} e^{-\lambda_{1}(1-s)} P(u, v)(s) d s\right]\right\} \\
& +\int_{0}^{t} e^{-\lambda_{1}(t-s)} P(u, v)(s) d s \mid \\
\leq & A_{1}\left\{| a | ( \lambda _ { 2 } \xi - 1 + e ^ { - \lambda _ { 2 } \xi } ) \left[\left(m_{2}\|u\|+n_{2}\|v\|+N_{2}\right) \int_{0}^{1} e^{-\lambda_{2}(1-s)}(Q \mathbf{1})(s) d s\right.\right. \\
& \left.+|b|\left(m_{1}\|u\|+n_{1}\|v\|+N_{1}\right) \int_{0}^{\eta} e^{-\lambda_{1}(\eta-s)}(P \mathbf{1})(s) d s\right] \\
& +\left(\lambda_{2}-1+e^{-\lambda_{2}}\right)\left[|a|\left(m_{2}\|u\|+n_{2}\|v\|+N_{2}\right) \int_{0}^{\xi} e^{-\lambda_{2}(\xi-s)}(Q \mathbf{1})(s) d s\right. \\
& \left.\left.+\left(m_{1}\|u\|+n_{1}\|v\|+N_{1}\right) \int_{0}^{1} e^{-\lambda_{1}(1-s)}(P \mathbf{1})(s) d s\right]\right\} \\
& +\left(m_{1}\|u\|+n_{1}\|v\|+N_{1}\right) \int_{0}^{t} e^{-\lambda_{1}(t-s)}(P \mathbf{1})(s) d s \\
\leq & \frac{A_{1}\left[|a b|\left(\lambda_{2} \xi-1+e^{-\lambda_{2} \xi}\right)\left(1-e^{-\lambda_{1} \eta}\right) \eta^{p-1}+\left|\left(\lambda_{2}-1+e^{-\lambda_{2}}\right)\right|\left(1-e^{-\lambda_{1}}\right)\right]+\left(1-e^{-\lambda_{1}}\right)}{\lambda_{1} \Gamma(p)} \\
& \times\left(m_{1}\|u\|+n_{1}\|v\|+N_{1}\right)+\left(m_{2}\|u\|+n_{2}\|v\|+N_{2}\right) \\
& \times \frac{A_{1}\left[|a|\left(\lambda_{2} \xi-1+e^{-\lambda_{2} \xi}\right)\left(1-e^{-\lambda_{2}}\right)+\left(\lambda_{2}-1+e^{-\lambda_{2}}\right)|a|\left(1-e^{-\lambda_{2} \xi}\right) \xi q^{q-1}\right]}{\lambda_{2} \Gamma(q)} \\
\leq & M_{1}\left[\left(m_{1}+n_{1}\right) r+N_{1}\right]+M_{2}\left[\left(m_{2}+n_{2}\right) r+N_{2}\right] .
\end{aligned}
$$

Hence

$$
\left\|T_{1}(u, v)\right\| \leq M_{1}\left[\left(m_{1}+n_{1}\right) r+N_{1}\right]+M_{2}\left[\left(m_{2}+n_{2}\right) r+N_{2}\right]
$$

Similarly, for any $(u, v) \in B_{r}$, proceeding as in (3.6), we can get

$$
\left\|T_{2}(u, v)\right\| \leq M_{1}^{\prime}\left[\left(m_{1}+n_{1}\right) r+N_{1}\right]+M_{2}^{\prime}\left[\left(m_{2}+n_{2}\right) r+N_{2}\right] .
$$

Consequently,

$$
\begin{aligned}
\|T(u, v)\|_{1}= & \left\|T_{1}(u, v)\right\|+\left\|T_{2}(u, v)\right\| \\
\leq & M_{1}\left[\left(m_{1}+n_{1}\right) r+N_{1}\right]+M_{2}\left[\left(m_{2}+n_{2}\right) r+N_{2}\right] \\
& +M_{1}^{\prime}\left[\left(m_{1}+n_{1}\right) r+N_{1}\right]+M_{2}^{\prime}\left[\left(m_{2}+n_{2}\right) r+N_{2}\right] \\
\leq & r .
\end{aligned}
$$

Now for $\left(u_{1}, v_{1}\right),\left(u_{2}, v_{2}\right) \in X \times X$, and for any $t \in[0,1]$, we have

$$
\begin{aligned}
& \left|T_{1}\left(u_{2}, v_{2}\right)(t)-T_{1}\left(u_{1}, v_{1}\right)(t)\right| \\
& \leq \mid A_{1}(t)\left\{a ( \lambda _ { 2 } \xi - 1 + e ^ { - \lambda _ { 2 } \xi } ) \left[\int_{0}^{1} e^{-\lambda_{2}(1-s)}\left[Q\left(u_{2}, v_{2}\right)(s)-Q\left(u_{1}, v_{1}\right)(s)\right] d s\right.\right. \\
& \left.\quad-b \int_{0}^{\eta} e^{-\lambda_{1}(\eta-s)}\left[P\left(u_{2}, v_{2}\right)(s)-P\left(u_{1}, v_{1}\right)(s)\right] d s\right]
\end{aligned}
$$




$$
\begin{aligned}
& -\left(\lambda_{2}-1+e^{-\lambda_{2}}\right)\left[a \int_{0}^{\xi} e^{-\lambda_{2}(\xi-s)}\left[Q\left(u_{2}, v_{2}\right)(s)-Q\left(u_{1}, v_{1}\right)(s)\right] d s\right. \\
& \left.\left.-\int_{0}^{1} e^{-\lambda_{1}(1-s)}\left[P\left(u_{2}, v_{2}\right)(s)-P\left(u_{1}, v_{1}\right)(s)\right] d s\right]\right\} \\
& +\int_{0}^{t} e^{-\lambda_{1}(t-s)}\left[P\left(u_{2}, v_{2}\right)(s)-P\left(u_{1}, v_{1}\right)(s)\right] d s \mid \\
\leq & A_{1}\left\{| a | ( \lambda _ { 2 } \xi - 1 + e ^ { - \lambda _ { 2 } \xi } ) \left[\left(m_{2}\left\|u_{2}-u_{1}\right\|+n_{2}\left\|v_{2}-v_{1}\right\|\right) \int_{0}^{1} e^{-\lambda_{2}(1-s)}(Q \mathbf{1})(s) d s\right.\right. \\
& \left.+|b|\left(m_{1}\left\|u_{2}-u_{1}\right\|+n_{1}\left\|v_{2}-v_{1}\right\|\right) \int_{0}^{\eta} e^{-\lambda_{1}(\eta-s)}(P \mathbf{1})(s) d s\right] \\
& +\left(\lambda_{2}-1+e^{-\lambda_{2}}\right)\left[|a|\left(m_{2}\left\|u_{2}-u_{1}\right\|+n_{2}\left\|v_{2}-v_{1}\right\|\right) \int_{0}^{\xi} e^{-\lambda_{2}(\xi-s)}(Q \mathbf{1})(s) d s\right. \\
& \left.\left.+\left(m_{1}\left\|u_{2}-u_{1}\right\|+n_{1}\left\|v_{2}-v_{1}\right\|\right) \int_{0}^{1} e^{-\lambda_{1}(1-s)}(P \mathbf{1})(s) d s\right]\right\} \\
\leq & M_{1}\left(m_{1}\left\|u_{2}-u_{1}\right\|+n_{1}\left\|v_{2}-v_{1}\right\|\right)+M_{2}\left(m_{2}\left\|u_{2}-u_{1}\right\|+n_{2}\left\|v_{2}-v_{1}\right\|\right) \\
& +\left(m_{1}\left(m_{1}+n_{1}\right)+M_{2}\left(m_{2}+n_{2}-u_{1}\right)\right]\left(\left\|u_{2}-u_{1}\right\|+\left\|v_{2}-v_{1}\right\|\right) . \\
\leq & \frac{A_{1}\left[|a b|\left(\lambda_{2} \xi-1+n_{1}\left\|v_{2}-v_{1}\right\|\right) \int_{0}^{t} e^{-\lambda_{1}(t-s)}(P \mathbf{1})(s) d s\right.}{A_{1}\left[|a|\left(\lambda_{2} \xi-1+e^{-\lambda_{1} \eta}\right) \eta^{p-1}+\left(\lambda_{2}-1+e^{-\lambda_{2}}\right)\left(1-e^{-\lambda_{1}}\right)\right]+\left(1-e^{-\lambda_{1}}\right)} \\
& \left.\times\left(m_{1}\left\|u_{2}-u_{1}\right\|+n_{1}\left\|v_{2}-v_{1}\right\|\right)+\left(m_{2}\left\|u_{2}-u_{1}\right\|+n_{2}\left\|v_{2}-v_{1}\right\|\right)+\left(\lambda_{2}-1+e^{-\lambda_{2}}\right)|a|\left(1-e^{-\lambda_{2} \xi}\right) \xi q-1\right] \\
& +(q)
\end{aligned}
$$

Consequently, for $\left(u_{1}, v_{1}\right),\left(u_{2}, v_{2}\right) \in X \times X$, we obtain

$$
\begin{aligned}
& \left\|T_{1}\left(u_{2}, v_{2}\right)-T_{1}\left(u_{1}, v_{1}\right)\right\| \\
& \quad \leq\left[M_{1}\left(m_{1}+n_{1}\right)+M_{2}\left(m_{2}+n_{2}\right)\right]\left(\left\|u_{2}-u_{1}\right\|+\left\|v_{2}-v_{1}\right\|\right) .
\end{aligned}
$$

Similarly, for $\left(u_{1}, v_{1}\right),\left(u_{2}, v_{2}\right) \in X \times X$, we can obtain

$$
\begin{aligned}
& \left\|T_{2}\left(u_{2}, v_{2}\right)-T_{2}\left(u_{1}, v_{1}\right)\right\| \\
& \quad \leq\left[M_{1}^{\prime}\left(m_{1}+n_{1}\right)+M_{2}^{\prime}\left(m_{2}+n_{2}\right)\right]\left(\left\|u_{2}-u_{1}\right\|+\left\|v_{2}-v_{1}\right\|\right) .
\end{aligned}
$$

It follows from (3.7) and (3.8) that

$$
\begin{aligned}
& \left\|T\left(u_{2}, v_{2}\right)-T\left(u_{1}, v_{1}\right)\right\|_{1} \\
& \quad \leq\left[\left(M_{1}+M_{1}^{\prime}\right)\left(m_{1}+n_{1}\right)+\left(M_{2}+M_{2}^{\prime}\right)\left(m_{2}+n_{2}\right)\right]\left(\left\|u_{2}-u_{1}\right\|+\left\|v_{2}-v_{1}\right\|\right) .
\end{aligned}
$$

Since $\left(M_{1}+M_{1}^{\prime}\right)\left(m_{1}+n_{1}\right)+\left(M_{2}+M_{2}^{\prime}\right)\left(m_{2}+n_{2}\right)<1, T$ is a contraction operator. So, by the fixed point theorem of the contraction mapping principle, the operator $T$ has a unique fixed point, which is the unique solution of the system (1.1). The proof is complete. 


\section{Applications}

Example 4.1 Consider the fractional differential system

$$
\begin{cases}{ }^{\mathrm{c}} D^{\frac{5}{2}}(D+2) u(t)=f_{1}(t, u(t), v(t)), & 0<t<1, \\ { }^{\mathrm{c}} D^{\frac{9}{4}}(D+3) v(t)=f_{2}(t, u(t), v(t)), & 0<t<1, \\ u(0)=u^{\prime}(0)=0, \quad u(1)=a v(\xi), & \\ v(0)=v^{\prime}(0)=0, \quad v(1)=b u(\eta), & \end{cases}
$$

where $\lambda_{1}=2, \lambda_{2}=3$ are two positive parameters. We take $a=\frac{2}{3}, b=3 e, \xi=\frac{1}{3}, \eta=\frac{1}{2}$.

By direct calculation, we have

$$
\begin{aligned}
& \Lambda \approx-1.5914367 \neq 0, \\
& A_{1} \approx 0.71340273, \quad A_{2} \approx 1.28801043, \\
& M_{1} \approx 0.92074193, \quad M_{2} \approx 0.09483186, \\
& M_{1}^{\prime} \approx 2.25908866, \quad M_{2}^{\prime} \approx 0.80969458 .
\end{aligned}
$$

Let

$$
\begin{aligned}
& f_{1}(t, x, y)=\frac{t}{t^{2}+1}\left(2+\frac{1}{10} \sin x+\frac{1}{8} \cos ^{2} y\right), \quad t \in[0,1], x, y \in \mathbb{R}, \\
& f_{2}(t, x, y)=\frac{1}{(t+2)^{2}}(2+x+2 \sin y), \quad t \in[0,1], x, y \in \mathbb{R} .
\end{aligned}
$$

Notice that

$$
\begin{aligned}
& \left|f_{1}(t, x, y)\right|=\left|\frac{t}{t^{2}+1}\left(2+\frac{1}{10} \sin x+\frac{1}{8} \cos ^{2} y\right)\right| \leq 2+\frac{1}{10}|x|+\frac{1}{8}|y|, \\
& \left|f_{2}(t, x, y)\right|=\left|\frac{1}{(t+2)^{2}}(2+x+2 \sin y)\right| \leq \frac{1}{2}+\frac{1}{4}|x|+\frac{1}{2}|y|,
\end{aligned}
$$

and

$$
\begin{aligned}
& \left(M_{1}+M_{1}^{\prime}\right) \rho_{1}+\left(M_{2}+M_{2}^{\prime}\right) \rho_{2} \approx 0.54411467<1 \\
& \left(M_{1}+M_{1}^{\prime}\right) \delta_{1}+\left(M_{2}+M_{2}^{\prime}\right) \delta_{2} \approx 0.84974204<1
\end{aligned}
$$

Therefore, all conditions of Theorem 3.1 are satisfied, and hence by Theorem 3.1 the system (4.1) has at least one solution.

Example 4.2 In Example 4.1, we only change $f_{1}, f_{2}$, and keep the other conditions unchanged. $\Lambda, A_{1}, A_{2}, M_{1}, M_{2}, M_{1}^{\prime}, M_{2}^{\prime}$ are as in (4.2). Let

$$
\begin{aligned}
& f_{1}(t, x, y)=\frac{t}{18(t+1)^{2}} x+\frac{1}{9} \arctan y, \quad t \in[0,1], x, y \in \mathbb{R}, \\
& f_{2}(t, x, y)=-\frac{1}{8} \cos x+\frac{1}{12} \sin y, \quad t \in[0,1], x, y \in \mathbb{R} .
\end{aligned}
$$


Noticing

$$
\begin{aligned}
\left|f_{1}\left(t, x_{1}, y_{1}\right)-f_{1}\left(t, x_{2}, y_{2}\right)\right| & \leq \frac{1}{18}\left|x_{1}-x_{2}\right|+\frac{1}{9}\left|y_{1}-y_{2}\right|, \\
\left|f_{2}\left(t, x_{1}, y_{1}\right)-f_{2}\left(t, x_{2}, y_{2}\right)\right| & \leq \frac{1}{8}\left|x_{1}-x_{2}\right|+\frac{1}{12}\left|y_{1}-y_{2}\right|,
\end{aligned}
$$

and

$$
\left(M_{1}+M_{1}^{\prime}\right)\left(m_{1}+n_{1}\right)+\left(M_{2}+M_{2}^{\prime}\right)\left(m_{2}+n_{2}\right) \approx 0.71841477<1 .
$$

Thus all conditions of Theorem 3.2 are satisfied and, consequently, the system (4.1) has a unique solution (with $f_{1}, f_{2}$ as in (4.3)).

\section{Competing interests}

The authors declare that they have no competing interests.

\section{Authors' contributions}

All authors contributed equally to the writing of this paper. All authors read and approved the final manuscript.

\section{Acknowledgements}

The authors would like to thank the referee for his/her very important comments, which improved the results and the quality of the paper. This work was supported financially by the National Natural Science Foundation of China (11371221), the Natural Science Foundation of Shandong Province of China (ZR2014AL004, ZR2013AQ014) and the Project of Shandong Province Higher Educational Science and Technology Program (J14LI08, J13LI08), Doctoral Scientific Research Foundation of Qufu Normal University and Youth Foundation of Qufu Normal University (BSQD20130140).

Received: 7 March 2016 Accepted: 16 August 2016 Published online: 30 August 2016

\section{References}

1. Culshaw, R, Ruan, S: A delay-differential equation model of HIV infection of CD4+ T-cells. Math. Biosci. 165, 27-39 (2000)

2. Nelson, P, Perelson, A: Mathematical analysis of delay differential equation models of HIV-1 infection. Math. Biosci. 179, $73-94$ (2002)

3. Tuckwell, H, Wan, F: On the behavior of solutions in viral dynamical models. Biosystems 73, 157-161 (2004)

4. Wang, L, Li, M: Mathematical analysis of the global dynamics of a model for HIV infection of CD4 ${ }^{+} \mathrm{T}$ cells. Math. Biosci. 200, 44-57 (2006)

5. Perelson, A: Modeling the interaction of the immune system with HIV. In: Castillo-Chavez, C (ed.) Mathematical and Statistical Approaches to AIDS Epidemiology. Lecture Notes in Biomathematics, vol. 83, pp. 350-370. Springer, New York (1989)

6. Perelson, A, Kirschner, D, Boer, R: Dynamics of HIV infection of CD4+ T cells. Math. Biosci. 114, 81-125 (1993)

7. Arafal, AAM, Rida, SZ, Khalil, M: Fractional modeling dynamics of HIV and CD4+ ${ }^{+}$-cells during primary infection. Nonlinear Biomed. Phys. 6, 1 (2012)

8. Cole, K: Electric conductance of biological systems. In: Proc. Cold Spring Harbor Symp. Quant. Biol., pp. 107-116. Cold Spring Harbor Laboratory Press, New York (1993)

9. Djordjević, V, Jarić, J, Fabry, B, Fredberg, J, Stamenović, D: Fractional derivatives embody essential features of cell rheological behavior. Ann. Biomed. Eng. 31, 692-699 (2003)

10. Agmon, S, Douglis, A, Nirenberg, L: Estimates near the boundary for solutions of elliptic partial differential equations satisfying general boundary conditions II. Commun. Pure Appl. Math. 17, 35-92 (1964)

11. Amann, H: Parabolic evolution equations and nonlinear boundary conditions. J. Differ. Equ. 72, $201-269$ (1988)

12. Aronson, D: A comparison method for stability analysis of nonlinear parabolic problems. SIAM Rev. 20, $245-264$ (1978)

13. Cardanobile, S, Mugnolo, D: Parabolic systems with coupled boundary conditions. J. Differ. Equ. 247, 1229-1248 (2009)

14. Delgado, M, Morales-Rodrigo, C, Suárez, A, Tello, J: On a parabolic-elliptic chemotactic model with coupled boundary conditions. Nonlinear Anal., Real World Appl. 11, 3884-3902 (2010)

15. Ferreira, R, Pablo, A, Quiros, F, Rossi, J: Non-simultaneous quenching in a system of heat equations coupled at the boundary. Z. Angew. Math. Phys. 57, 586-594 (2006)

16. Leung, A: A semilinear reaction-diffusion prey-predator system with nonlinear coupled boundary conditions: equilibrium and stability. Indiana Univ. Math. J. 31, 223-241 (1982)

17. Mikhailov, M: General solutions of the diffusion equations coupled at boundary conditions. Int. J. Heat Mass Transf. 16, 2155-2164 (1973)

18. Pao, C: Finite difference reaction-diffusion systems with coupled boundary conditions and time delays. J. Math. Anal. Appl. 272, 407-434 (2002)

19. Rossi, J: The blow-up rate for a system of heat equations with non-trivial coupling at the boundary. Math. Methods Appl. Sci. 20, 1-11 (1997) 
20. Wang, S: Doubly nonlinear degenerate parabolic systems with coupled nonlinear boundary conditions. J. Differ. Equ. $182,431-469$ (2002)

21. Ahmad, B, Alsaedi, A: Existence and uniqueness of solutions for coupled systems of higher-order nonlinear fractional differential equations. Fixed Point Theory Appl. 2010, Article ID 364560 (2010)

22. Ahmad, B, Nieto, J: Existence results for a coupled system of nonlinear fractional differential equations with three-point boundary conditions. Comput. Math. Appl. 58, 1838-1843 (2009)

23. Ahmad, B, Ntouyas, SK: Existence results for a coupled system of Caputo type sequential fractional differential equations with nonlocal integral boundary conditions. Appl. Math. Comput. 266, 615-622 (2015)

24. Ahmad, B, Ntouyas, S, Alsaedi, A: On a coupled system of fractional differential equations with coupled nonlocal and integral boundary conditions. Chaos Solitons Fractals 83, 234-241 (2016)

25. Aljoudi, S, Ahmad, B, Nieto, J, Alsaedi, A: A coupled system of Hadamard type sequential fractional differential equations with coupled strip conditions. Chaos Solitons Fractals 91, 39-46 (2016)

26. Bai, C, Fang, J: The existence of a positive solution for a singular coupled system of nonlinear fractional differential equations. Appl. Math. Comput. 150, 611-621 (2004)

27. Goodrich, C: Existence of a positive solution to systems of differential equations of fractional order. Comput. Math. Appl. 62, 1251-1268 (2011)

28. Jiang, W: Solvability for a coupled system of fractional differential equations at resonance. Nonlinear Anal., Real World Appl. 13, 2285-2292 (2012)

29. Rehman, M, Khan, R: A note on boundary value problems for a coupled system of fractional differential equations. Comput. Math. Appl. 61, 2630-2637 (2011)

30. Su, X: Boundary value problem for a coupled system of nonlinear fractional differential equations. Appl. Math. Lett. $22,64-69(2009)$

31. Yuan, C: Two positive solutions for $(n-1,1)$-type semipositone integral boundary value problems for coupled systems of nonlinear fractional differential equations. Commun. Nonlinear Sci. Numer. Simul. 17, 930-942 (2012)

32. Wang, J, Xiang, H, Liu, Z: Positive solution to nonzero boundary values problem for a coupled system of nonlinear fractional differential equations. Int. J. Differ. Equ. 2010, Article ID 186928 (2010)

33. Kilbas, AA, Srivastava, HM, Trujillo, JJ: Theory and Applications of Fractional Differential Equations. Elsevier, Amsterdam (2006)

34. Podlubny, l: Fractional Differential Equations. Academic Press, San Diego (1999)

35. Alsaedi, A, Ntouyas, S, Agarwal, R, Ahmad, B: On Caputo type sequential fractional differential equations with nonlocal integral boundary conditions. Adv. Differ. Equ. 2015, 33 (2015)

36. Grans, A, Dugundji, J: Fixed Point Theorems. Springer, New York (2005)

\section{Submit your manuscript to a SpringerOpen ${ }^{\circ}$ journal and benefit from:}

- Convenient online submission

Rigorous peer review

- Immediate publication on acceptance

- Open access: articles freely available online

- High visibility within the field

- Retaining the copyright to your article 